\title{
Pituitary incidentaloma diagnosed as acromegaly triggered by trauma: A case report
}

\author{
Yusuke Morinaga $^{1, *}$, Ichiro Abe ${ }^{2}$, Kimiya Sakamoto ${ }^{1}$, Ritsurou Inoue ${ }^{1}$, Takafumi Mitsutake ${ }^{1}$, \\ Hayatsura Hanada ${ }^{1}$, Seiji Haraoka ${ }^{3}$, Kouhei Nii ${ }^{1}$ \\ ${ }^{1}$ Department of Neurosurgery, Fukuoka University Chikushi Hospital, Chikushino City, Fukuoka, Japan; \\ ${ }^{2}$ Department of Endocrinology and Diabetes Mellitus, Fukuoka University Chikushi Hospital, Chikushino City, Fukuoka, Japan; \\ ${ }^{3}$ Department of Pathology, Fukuoka University Chikushi Hospital, Chikushino City, Fukuoka, Japan.
}

\begin{abstract}
Summary Pituitary incidentaloma (PI) is a generic term for pituitary tumors that are identified on images acquired for non-malignant conditions. Acromegaly is an extremely rare form of PI. Occasionally, a functional pituitary adenoma (PA) may be misdiagnosed as PI, which may result in a poor clinical outcome. Here we report the first case, to the best of our knowledge, of PI diagnosed as trauma-triggered acromegaly. A 42-year-old man with a chief complaint of head trauma was referred to our hospital after computed tomography (CT) revealed a pituitary tumor. His appearance was suggestive of acromegaly. Mild hypertrophy of the extremities was also observed. Preoperative blood tests, magnetic resonance imaging (MRI), and endocrine tolerance test findings indicated acromegaly. Accordingly, we suspected a growth hormone (GH)-producing $\mathrm{PA}$, and we performed endoscopic transsphenoidal surgery (eTSS). Histopathology showed a densely granulated GH-producing PA, which was also confirmed via immunohistochemistry. Two months after surgery, blood tests showed decreased levels of GH and insulin-like growth factor-1. In addition, a postoperative endocrine tolerance test revealed no abnormalities. There was no recurrence at 24 months after surgery. The findings from this case suggest that PIs can also present as functional adenomas, which can be diagnosed using initial hormone examinations and endocrine tolerance tests. Therefore, thorough endocrine examination is necessary for early diagnosis and treatment and improved patient outcomes.
\end{abstract}

Keywords: Acromegaly, pituitary incidentaloma, trauma

\section{Introduction}

Pituitary incidentaloma (PI) is a generic term for pituitary tumors that are first identified on images acquired for reasons unrelated to tumors, such as headache, trauma, or symptoms involving the neck or central nervous system (1-4). The wide application of sensitive brain imaging techniques such as computed tomography $(\mathrm{CT})$ and magnetic resonance imaging (MRI) has led to increased recognition of such lesions. Although the etiology of PIs covers a wide range of

*Address correspondence to:

Dr. Yusuke Morinaga, Department of Neurosurgery, Fukuoka University Chikushi Hospital, 1-1-1 Zokumyoin, Chikushino City, Fukuoka Prefecture 818-8502, Japan.

E-mail: yu_the_morio@yahoo.co.jp pathologies [most of them (approximately 90\%) are benign adenomas], they may result in visual and/or neurological abnormalities.

By definition, micro-incidentalomas have a maximum diameter of $<1 \mathrm{~cm}$, while the diameter of macro-incidentalomas is at least $1 \mathrm{~cm}$. Microincidentalomas have a reported mean prevalence of approximately $10 \%$ in normal individuals (1). Among PIs, growth hormone (GH)-producing pituitary adenomas (PAs) and acromegaly are extremely rare, with a prevalence of only $0.14 \%$ (2). Acromegaly is a rare disease, largely caused by GH-producing pituitary adenomas. However, its incidence is higher than previously believed, and early diagnosis is essential as there is an increased risk of morbidity and mortality when inappropriate treatment is given. Screening is recommended for all patients with clinical features of 
Table 1. Blood and endocrine test results for a 42-yearold man with a pituitary incidentaloma diagnosed as acromegaly triggered by trauma

\begin{tabular}{ll}
\hline white blood cell count & $5.6 \times 10^{3} / \mu \mathrm{L}$ \\
red blood cell count & $495 \times 10^{4} / \mu \mathrm{L}$ \\
hemoglobin & $14.6 \mathrm{~g} / \mathrm{dL}$ \\
hematocrit & $45.7 \%$ \\
platelet count & $25.2 \times 10^{4} / \mu \mathrm{L}$ \\
$\mathrm{Na}$ & $141 \mathrm{mmol} / \mathrm{L}$ \\
$\mathrm{K}$ & $3.8 \mathrm{mmol} / \mathrm{L}$ \\
$\mathrm{Cl}$ & $10^{4} \mathrm{mmol} / \mathrm{L}$ \\
$\mathrm{HbA1c}$ & $6.0 \%$ \\
$\mathrm{LDL}$ & $86 \mathrm{mg} / \mathrm{dL}$ \\
$\mathrm{C}-$-reactive protein & $0.09 \mathrm{mg} / \mathrm{dL}$ \\
D-dimers & $<0.3 \mu \mathrm{gg} / \mathrm{mL}$ \\
& \\
Endocrine tests: & \\
growth hormone (GH) & $4.97 \mathrm{ng} / \mathrm{mL}$ \\
insulin-like growth factor-1 (IGF-1) & $426 \mathrm{ng} / \mathrm{mL}$ \\
luteinizing hormone (LH) & $1.76 \mathrm{mIU} / \mathrm{mL}$ \\
follicle-stimulating hormone (FSH) & $3.70 \mathrm{mIU} / \mathrm{mL}$ \\
adrenocorticotropic hormone & $20.4 \mathrm{pg} / \mathrm{mL}$ \\
prolactin (PRL) & $8.46 \mathrm{ng} / \mathrm{m}$ \\
anti-diuretic hormone & $0.8 \mathrm{pg} / \mathrm{mL}$ \\
thyroid-stimulating hormone (TSH) & $1.08 \mu \mathrm{IU} / \mathrm{mL}$ \\
free thyroxine (FT4) & $1.05 \mathrm{ng} / \mathrm{dL}$ \\
free triiodothyronine (FT3) & $17.9 \mathrm{pg} / \mathrm{L}$ \\
cortisol & $6.96 \mu \mathrm{gg} / \mathrm{dL}$. \\
\hline
\end{tabular}

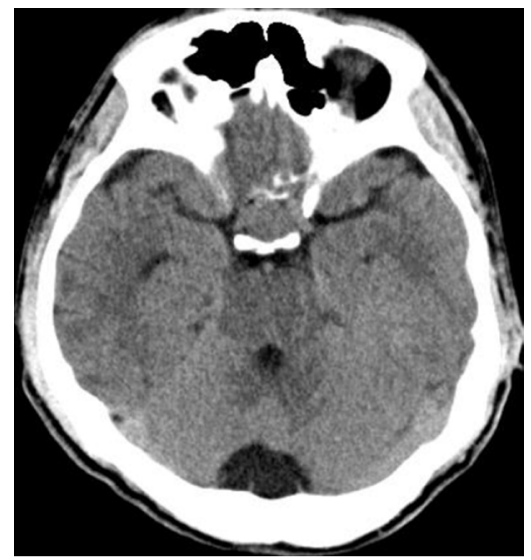

Figure 1. Brain computed tomography (CT) findings at initial presentation of a 42 -year-old man with a pituitary incidentaloma. The image shows a pituitary tumor in the sella. excess GH production. There is increasing knowledge that the classical acromegaly diagnostic criteria can no longer be applied broadly to everyone; some patients can have GH excess with a normal GH response to glucose. Treatment is multifactorial, and personalized therapy is thus advised (5).

Here, we report the first case, to our knowledge, of PI diagnosed as trauma-triggered acromegaly and present a review of the relevant literature.

\section{Case Report}

A 42-year-old man presented with a chief complaint of head trauma. He had no past history of tumors, head trauma, or other factors related to the development of a pituitary tumor. We performed head CT, which identified a pituitary tumor. He was admitted to our hospital in a fully conscious condition. His blood pressure and body temperature were $110 / 62 \mathrm{mmHg}$ and $36.2^{\circ} \mathrm{C}$, respectively. His height and weight were $170 \mathrm{~cm}$ and $64.5 \mathrm{~kg}$, respectively. Heart and respiratory sounds were normal, and there were no neurological deficits. His heart rate was 62 beats/min. He exhibited an acromegaly-like appearance, and there were no obvious differences between his current appearance and that on his driver's license photograph taken 5 years prior. He also exhibited mild hypertrophy of the extremities, although there was no tongue enlargement or complaint of sleep apnea.

Blood and endocrine test results are outlined in Table 1. Electrocardiography and upper and lower gastrointestinal examinations revealed no abnormal findings. Head CT showed a slightly dense tumorous lesion that progressed from the intrasellar to the suprasellar regions (Figure 1). Radiography showed mandibular protrusion, flower cabbage-like deformities in the distal phalanges of the fingers, and 24-mm-thick soft tissue on the bilateral heel pads (Figure 2A, B, C). Brain MRI showed a tumorous lesion on the pituitary gland (maximum size: $22 \mathrm{~mm}$ ), which exhibited a strong signal on gadolinium (Gd)-enhanced T1-
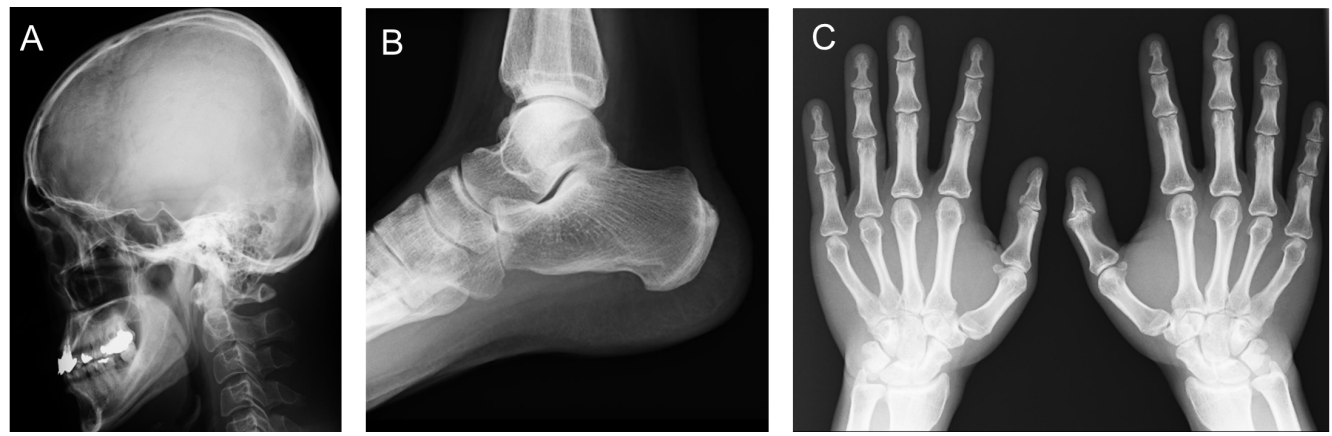

Figure 2. Morphological characteristics observed by radiography for a 42-year-old man with a pituitary incidentaloma. (A) A lateral skull radiograph shows mandibular protrusion, occipital nodule protrusion, skull thickening, and ballooning of the sella. (B) A foot radiograph shows thickening of the heel pad. (C) A hand radiograph shows lower cabbage-like, hypertrophic deformities in the distal phalanges. 
A

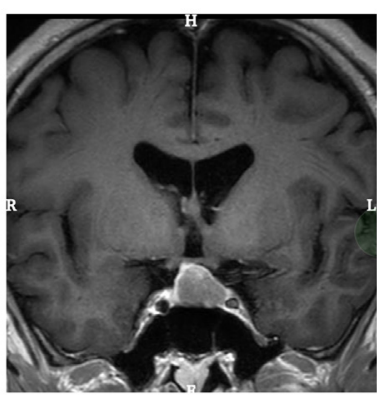

B

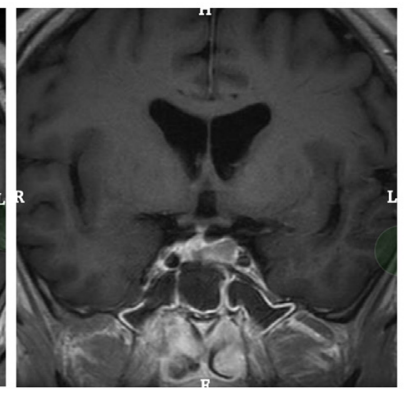

Figure 3. Gadolinium-enhanced T1-weighted magnetic resonance imaging (MRI) findings of a 42-year-old man with a pituitary incidentaloma. (A) An image obtained at presentation showing a pituitary tumor. (B) A postoperative image showing complete disappearance of the tumor.

weighted imaging (T1WI; Figure 3A).

\subsection{Preoperative endocrine tolerance test}

The corticotropin-releasing hormone $(\mathrm{CRH})$ loading test revealed a paradoxical reaction of GH. The thyroid releasing hormone (TRH) loading test also yielded a paradoxical reaction of $\mathrm{GH}$ and a normal reaction of thyroid stimulating hormone (TSH). The luteinizing hormone-releasing hormone (LH-RH) loading test yielded normal reactions of $\mathrm{GH}$, while the growth hormone releasing peptide-2 (GHRP-2) loading test yielded a normal reaction of GH and prolactin (PRL). The 75-g oral glucose tolerance loading test yielded a paradoxical reaction of $\mathrm{GH}$, indicating the absence of diabetes. The bromocriptine and octreotide loading tests yielded a paradoxical decline in $\mathrm{GH}$.

\subsection{Post-hospitalization course}

The patient's symptoms satisfied the diagnostic criteria for acromegaly, and endoscopic transsphenoidal surgery (eTSS) was performed. After incising the dura mater at the bottom of the sella, we performed extracapsular extraction followed by gross total resection. No cerebrospinal fluid leakage was observed during eTSS, and the surgery was completed after reconstruction of the bottom of the sella. Permanent pathology revealed a densely granulated, GH-producing PA, and immunohistology revealed GH-positive intracellular staining (Figure 4A, B).

\subsection{Postoperative course}

After surgery, we did not observe recurrence of the tumor or intraoperative hemostasis on MRI (Figure 3B). There were no complications such as cerebrospinal fluid leakage or diabetes insipidus. The patient was prescribed hydrocortisone $(15 \mathrm{mg} /$ day) and was discharged. Two months later, he underwent an endocrine tolerance test, which yielded GH and IGF-1 levels of 0.41 and $156 \mathrm{ng} /$
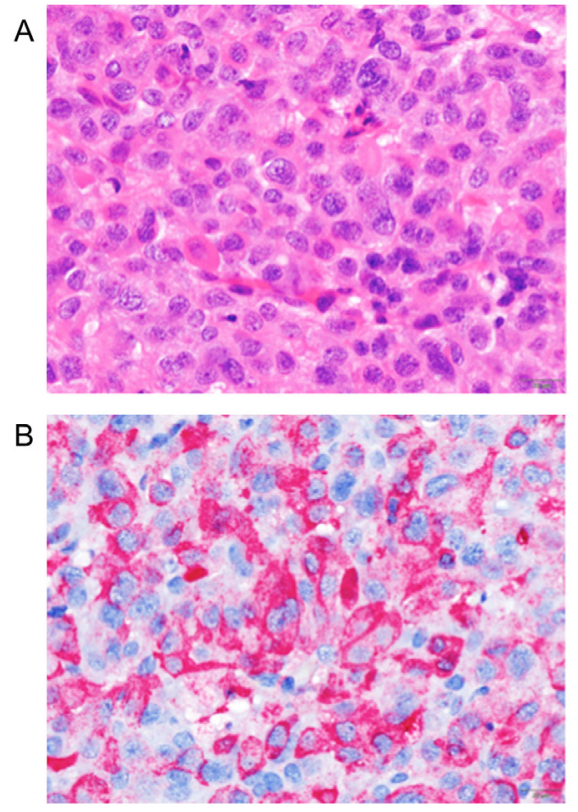

Figure 4. (A) Permanent pathology reveals a densely granulated, GH-producing PA (hematoxylin and eosin stain; H\&E $\times 60)$, and (B) immunohistology reveals GHpositive intracellular staining $(\mathrm{GH} \times 60)$.

$\mathrm{mL}$, respectively. Thus, the patients' hormone levels had normalized relative to preoperative baseline levels. The CRH loading test yielded a paradoxical reaction of GH, while the TRH, LH-RH, and GHRP-2 loading tests yielded normal reactions. All other test results were normal. Twelve months after surgery, the patient underwent endocrine blood sampling which revealed further decreases in GH and IGF-1 levels (0.32 and $112 \mathrm{ng} / \mathrm{mL}$, respectively). MRI revealed no recurrence, and hydrocortisone administration was terminated. The patient is currently being followed as an outpatient.

\section{Discussion}

A GH-producing adenoma is classically diagnosed based on a combination of increased GH levels, unsuppressed GH levels after a $75-\mathrm{g}$ oral glucose tolerance test, and increased insulin-like growth factor (IGF)-1 levels. In our case, the 75 -g oral glucose tolerance test had a bottom value of 1 or more, and the patient was diagnosed with clinical acromegaly from the $75-\mathrm{g}$ oral glucose tolerance test and his acromegaly facial features.

Acromegaly can be promptly and easily diagnosed. The most common cause is a GH-producing PA, and the disease is characterized by facial features such as frontal bossing, thick eyelids, a large triangular nose, a thickened lower lip, and macroglossia; hypertrophy of the extremities; bone deformities; hyperhidrosis; and headache. Acromegaly is often also accompanied by impaired glucose tolerance, hypertension, metabolic disorders such as dyslipidemia and goiter, colon polyps, and malignant tumors (particularly colon cancer). Disease outcomes and life expectancy deteriorate 
when it is untreated; therefore, early diagnosis and treatment are crucial $(6,7)$. The patient in the current case was diagnosed with acromegaly on the basis of accentuated facial features, imaging findings, hormone tests, and endocrine tolerance tests. In a previous study that investigated 71 cases of PI, the overall mean age of patients was 51.6 years. The most common chief complaint was headache, and CT was used to detect the tumors (detection rate: 63.2\%). Moreover, 17.6\% patients had symptoms that facilitated early diagnosis. The diagnoses included pituitary adenoma (48 cases, 70.6\%), Rathke's cleft cyst (RCC; nine cases, 3.2\%), prolactinoma (five cases, 7\%), and GH-producing PA (one case, $1.4 \%$ ). In addition, 14 cases $(20 \%)$ were diagnosed after hormone evaluations. Twenty-one patients $(28.8 \%)$ underwent surgery according to the guidelines for PI treatment in Japan (8). This indicates that approximately $30 \%$ of PIs were indicated for surgery. Our patient was relatively young (42 years) and diagnosed with a GH-producing PA triggered by trauma, which is considered a rarity. Early diagnosis and treatment of PIs are important as functional adenomas, including acromegaly, may result in poor prognosis when there is a delay in diagnosis and treatment.

As far as we could find, based on a search of PubMed, this is the first case of PI diagnosed as acromegaly triggered by trauma (1-4). At the initial visit, the patient did not present symptoms suggesting PI or acromegaly. Early diagnosis and treatment resulted in complete remission in our case. Because PIs can become adaptive after surgery and patients can present with newly growing adenomas, regular endocrine tolerance tests, basal hormone value measurements, neurological and physical examinations, and visual acuity and visual field examinations during follow-up visits are essential. We also plan to monitor our patient over the long term.

In conclusion, the findings of this case suggest that
PIs can also present as functional adenomas, which can be initially diagnosed by hormone assessments and endocrine tolerance tests. Thus, it is important to perform these tests in case of abnormalities in basal hormone values. This will help in early diagnosis and treatment, which can ultimately improve patient outcomes.

\section{References}

1. Paschou SA, Vryonidou A, Goulis DG. Pituitary incidentalomas: A guide to assessment, treatment and follow-up. Maturitas. 2016; 92:143-149.

2. Sanno N, Oyama K, Tahara S, Teramoto A, Kato Y. A survey of pituitary incidentaloma in Japan. Eur J Endocrinol. 2003; 149:123-127.

3. Vaninetti NM, Clarke DB, Zwicker DA, Yip CE, Tugwell B, Doucette S, Theriault C, Aldahmani K, Imran SA. A comparative, population-based analysis of pituitary incidentalomas vs clinically manifesting sellar masses. Endocr Connect. 2018; 7:768-776.

4. Ogiwara T, Nagm A, Nakamura T, Mbadugha T, Horiuchi T, Hongo K. Significance and indications of surgery for asymptomatic nonfunctioning pituitary adenomas. World Neurosurg. 2019. pii: S1878-8750(19)31257-4.

5. Zahr R, Fleseriu M. Updates in diagnosis and treatment of acromegaly. Eur Edocrinol. 2018; 14:57-61.

6. Dekkers OM, Biermasz NR, Pereira AM, Romijn JA, Vandenbroucke JP. Mortality in acromegaly: A metaanalysis. J Clin Endocrinol Metab. 2008; 93:61-67.

7. Holdaway IM, Bolland MJ, Gamble GD. A meta-analysis of the effect of lowering serum levels of GH and IGF-I on mortality in acromegaly. Eur J Endocrinol. 2008; 159:8995.

8. Esteves C, Neves C, Augusto L, Menezes J, Pereira J, Bernardes I, Fonseca J, Carvalho D. Pituitary incidentalomas: analysis of a neuroradiological cohort. Pituitary. 2015; 18:777-781.

(Received June 11, 2019; Revised August 27, 2019; Accepted August 31, 2019) 